\title{
OpenMP and NUMA Architectures I: Investigating Memory Placement on the SGI Origin 3000
}

\author{
Nathan Robertson and Alistair Rendell \\ Department of Computer Science \\ Australian National University \\ Canberra ACT0200, Australia \\ Nathanr@nathanr.net, Alistair.Rendell@anu.edu.au
}

\begin{abstract}
The OpenMP programming model is based upon the assumption of uniform memory access. Virtually all current day large scale shared memory computers exhibit some degree of Non-Uniform Memory Access (NUMA). Should OpenMP be extended for NUMA architectures? This paper aims to quantify NUMA effects on the SGI Origin 3000 system as a prelude to answering this important question. We discuss the tools required to study NUMA effects and use them in the study of latency, bandwidth and the solution of a 2-D heat diffusion problem.
\end{abstract}

\section{Introduction}

In the late 1980's a number of hardware vendors began offering small scale shared memory parallel architectures comprising a few CPU and memory boards linked via a shared bus. These systems quickly proved to be very popular not least because the cost premium of adding CPUs was relatively low compared to the cost of the initial system. With this new architecture and an expanding user base came a natural interest to develop applications that could run in parallel, and this was particularly true among the computational science community.

Although there are several ways to utilize multiple CPUs on a shared memory architecture, the idea of parallelizing critical loops by adding a few directives to the source code and letting the compiler do most of the work was particularly attractive. This was in part because computational scientists had used a similar approach to successfully exploit vector architectures. To facilitate this, hardware vendors began implementing and promoting their own set of shared memory parallel programming compiler directives. Unfortunately, although many of these directives provided virtually identical functionality their exact form was vendor specific. This situation existed for a number of years, until in the mid 1990's there was a move to develop a standard set of shared memory parallel processing directives that eventually gave rise to OpenMP for Fortran 77 [1] (released in 1997). The success of this standardization effort has since led to a number of updates and extensions such that the OpenMP model now encompasses Fortran 95, C and C++ [1].

A basic premise of OpenMP is a shared memory programming model in which all CPUs have equal access to all memory locations, i.e. the assumption of Uniform Memory Access (UMA). While this accurately reflected the early bus based shared 
memory machines of the late 1980's and early 1990's, it is increasingly not true for large scale shared memory parallel architectures. Thus, virtually all current large scale parallel machines have some degree of Non Uniform Memory Access (NUMA), e.g. the SGI Origin 3000, HP GS320, Sun E15k and IBM Regatta.

For computational science the shared memory parallel programming paradigm has some definite attractions, especially now that there is widespread compiler support for OpenMP. For many of these applications, however, performance is also critical and an obvious question arises as to whether the existing UMA OpenMP programming model should be extended to account for current NUMA architectures, and if so how. There is also interest in developing OpenMP compilers that generate code for cluster systems [2,3] - which is an extreme NUMA environment!

NUMA support within OpenMP has received some discussion both in the literature and at various conference forums [4,5]. There are essentially two opposing views. At one extreme it is considered that issues relating to memory placement should be handled by the $\mathrm{O} / \mathrm{S}$ through sensible page placement and migration. While at the other extreme it is advocated that the user should be provided with full control over all memory and thread placement. In the middle there are a number of hybrid schemes, such as the user initiated page migration model proposed by Nicolopolous et al [6].

Already some vendors, e.g. SGI [7] and Compaq [8], have implemented their own NUMA extensions to OpenMP. Unfortunately, as in the early days of shared memory parallel systems, these have different syntax but similar functionality. For application programmers trying to develop portable programs this provides a major disincentive to their use. In our view, however, it is premature to be advocating any specific NUMA extensions to OpenMP. Rather we believe it is first important to gather information for a variety of applications showing clearly the benefits or otherwise of different memory placements on overall performance. That is, to quantify what is the maximum likely difference between the performance obtained when using optimal memory placement versus that obtained when using the worst possible memory placement, and then to compare both these results with the typical performance obtained when running with the default memory placement provided by the $\mathrm{O} / \mathrm{S}$.

This paper is a first step towards this goal. Firstly we discuss the basic functionality that is required to develop application programs that can be used to investigate NUMA effects. Then we use this functionality to measure the different memory latencies and bandwidths on a NUMA platform - an SGI Origin 3000 system $[9,10]$. Finally we investigate the implication of NUMA on the performance on the SGI Origin of a typical 2-D heat diffusion program.

\section{Basic Requirements}

Our goal is to place processes or threads on specific CPUs within the NUMA hardware and have their associated memory reside at specific physical locations. To achieve this requires the ability to:

- bind a particular process or thread to a specific CPU

- allocate memory pages to specific memory locations without page migration For verification it is also useful to be able to: 
- determine the physical CPU that a process/thread is currently running on

- map any given virtual address to its physical address

In general, in a multi-user shared-memory parallel environment having users tie their threads or memory to specific hardware resources is undesirable since it substantially reduces the flexibility of the $\mathrm{O} / \mathrm{S}$ to efficiently schedule jobs. Thus it is hardly surprising that for a given hardware platform finding out how to achieve these goals is not always easy!

The results presented here were obtained from an SGI Origin 3000 system [9]. To create threads we used the pthreads library and then bound them to specific CPUs using SGI's non-standard pthread_setrunon_np library routine. Memory was allocate and placed at specific locations using the "dplace" utility. To verify thread placement it is necessary to first issue a call to schedctl (SETHINTS) before obtaining the CPU id from the get_cpu macro defined in the task.h header file. To map virtual addresses to physical addresses we used the va2pa routine available at the SGI technical publication web site [11], although it should be noted that the bits are shifted by 33 places on the Origin 3000 compared to 32 places on the Origin 2000.

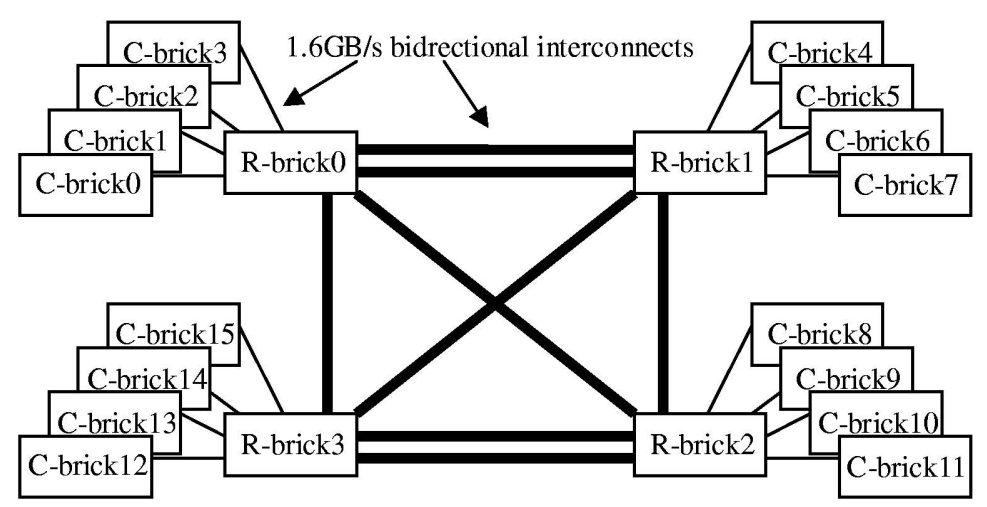

Fig. 1. Schematic diagram of the router and CPU bricks on the SGI Origin 3000 used in this work. (C-bricks contain 4 600MHz R14K CPUs and 4GBytes of memory).

The physical configuration of the SGI 3000 used in this work is given in Fig.1. It has 64 processors and 64GB of memory. Each CPU board, or C-brick, comprises 4 CPUs and 4GBytes of memory. The C-bricks are connected to router or R-bricks that are then connected together in a "bristled" hypercube topology. There are 8 connections to each R-brick and each is capable of a peak performance of $1.6 \mathrm{~GB} / \mathrm{s}$ bidirectional. On the machine used for this work the spare R-brick connections that would normally be used to build a higher dimensional hypercubes have been used to provided enhanced bandwidth between selected R-bricks. The configuration shown in Fig.1 has three different levels of NUMA, corresponding to the following memory accesses: 
1. within the same C-brick, e.g. C-brick0 to C-brick0

2. one R-brick remove, e.g. C-brick0 to C-brick1

3. two R-bricks removed, e.g. C-brick0 to C-brick11

\section{Measuring Memory Latency on NUMA Architectures}

The aim here is to measure the memory latency associated with each different class of memory access. To do this we have developed a modified version of LMBench [12]. The basic idea behind LMBench is pointer chasing, i.e. a memory location is read and the contents used as a pointer to the location of the next memory address to be read. By designing each memory reference to be sufficiently well separated from all previous references it is possible to ensure that all references are to main memory and not to data that already resides in cache.

To achieve the above goal LMBench was modified to use pthreads. First a thread is created and bound to a specific CPU. This thread then allocates and initializes a large block of memory on the same C-brick (using dplace). The first element of this array is defined as a pointer referencing an element $\mathrm{N}_{\text {sep }}$ bytes further along in the array. This element in turns references another element $\mathrm{N}_{\text {sep }}$ bytes further advanced. This pattern continues until after some point a null pointer is used to terminate the sequence. After establishing this pointer series, the address of every element in the array is examined to verify that it is indeed located on the expected Cbrick. The thread is then moved and bound to a CPU on a different C-brick, the pointer references followed, and the elapsed time taken recorded. (Note that we initialize the thread on one C-brick and then measure the pointer chasing time on another to ensure that that there are no caching effects resulting from the array setup phase). When measurement is complete on this C-brick the thread is moved to another C-brick and process repeated. A brief summary is given below:

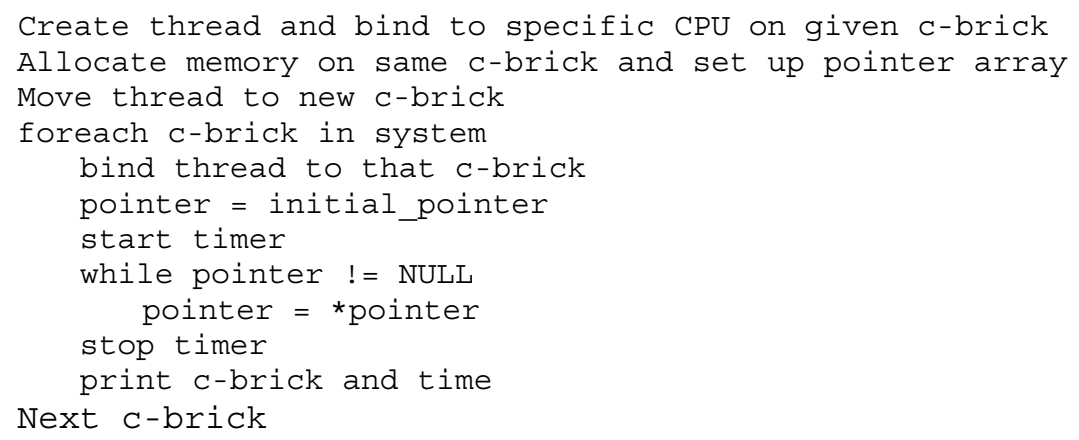

The benchmark contains two parameters, the number of bytes separating each pointer reference $\left(\mathrm{N}_{\text {sep }}\right)$ and the total number of pointer references $\left(\mathrm{N}_{\text {ref }}\right)$. The results for $\mathrm{N}_{\text {ref }}=1000$ and $\mathrm{N}_{\text {sep }}=16 \mathrm{kbytes}$ are given in Fig. 2. It is immediately obvious from this that the memory was located on C-brick 2. Accessing this memory from the C-bricks 
connected to the same R-brick (i.e. C-bricks 0,1 and 3 ) incurs a latency penalty of $\approx$ $50 \%$, while those located two R-bricks away have roughly twice the latency.

Overall the results shown in Fig. 1 tend to indicate that for memory latency critical applications there is potentially a factor of two to be gained through careful placement of thread and memory.

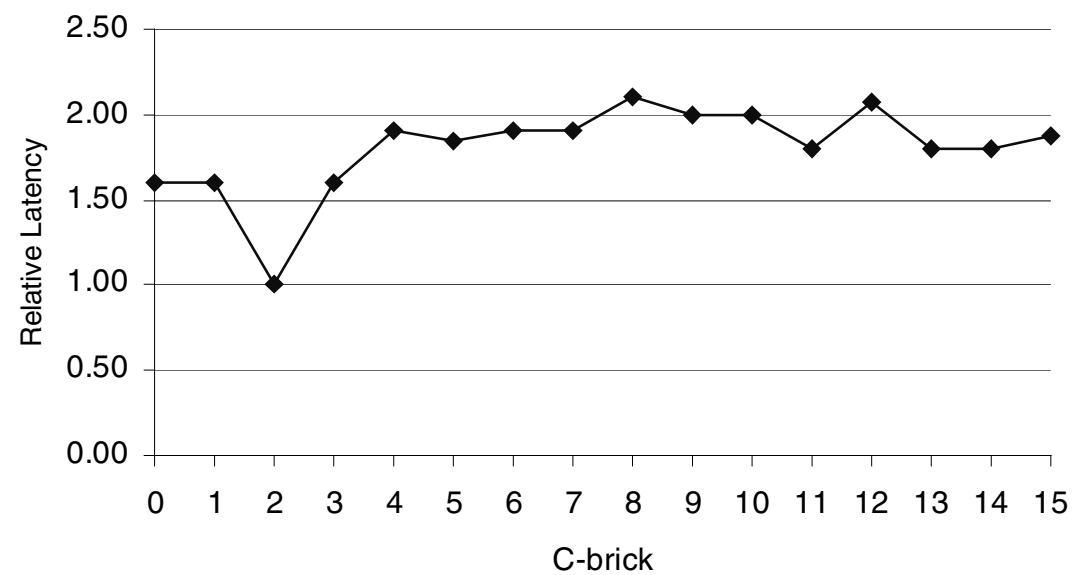

Fig. 2. Relative memory latency for accessing memory located on C-brick2 from all C-bricks. Results obtained on a non-dedicated Origin 3000

\section{Measuring Bandwidth on NUMA Architectures}

The aim here was to measure memory bandwidth contentions that may arise from having multiple threads simultaneously accessing various memory locations. To do this we have used a modified version of the STREAM benchmark [13]. This benchmark measures the time required to perform the following four vector operations:

$$
\begin{array}{lll}
A(1: N)=B(1: N) & \text { copy } \\
A(1: N)=B(1: N) * \text { const } & \text { scale } \\
A(1: N)=B(1: N)+C(1: N) & \text { add } \\
A(1: N)=B(1: N)+C(1: N) \text { * const triad }
\end{array}
$$

for some large value of $\mathrm{N}$. The results are reported in terms of Mbytes/sec, representing the amount of data that is transferred between memory and the CPU.

The basic STREAM benchmark was modified to create multiple threads and multiple sets of the A, B and C vectors. Each set of vectors was allocated entirely within the memory of a specific C-brick and was used by a unique thread. Treads 
were then selectively bound to different C-bricks and synchronized before and after they had completed each vector operation. The results obtained with up to two threads and two different memory locations are given in Table 1.

Table 1. Memory bandwidth for Scale and Triad operations with explicit thread and memory placement for up to 2 threads/memory pairs. Results were obtained from a non-dedicated 64 SGI O3K with $600 \mathrm{MHz}$ processors

\begin{tabular}{|c|c|c|c|c|c|c|}
\hline \multirow{2}{*}{$\begin{array}{l}\text { Exp. } \\
\text { No. }\end{array}$} & \multicolumn{2}{|c|}{ - C-brick Pair 1 - } & \multicolumn{2}{|c|}{ - C-brick Pair 2 - } & \multirow{2}{*}{$\begin{array}{c}\text { Scale } \\
\text { Mbytes/Sec }\end{array}$} & \multirow{2}{*}{$\begin{array}{c}\text { Triad } \\
\text { Mbytes/Sec }\end{array}$} \\
\hline & Thread & Memory & Thread & Memory & & \\
\hline \multicolumn{7}{|c|}{ 1-thread } \\
\hline 1.1 & 0 & 0 & - & - & 676 & 637 \\
\hline 1.2 & 0 & 1 & - & - & 556 & 533 \\
\hline 1.3 & 0 & 9 & - & - & 433 & 458 \\
\hline \multicolumn{7}{|c|}{ 2-threads } \\
\hline 2.1 & 0 & 0 & 0 & 0 & 789 & 800 \\
\hline 2.2 & 0 & 0 & 1 & 1 & 1196 & 1260 \\
\hline 2.3 & 0 & 1 & 1 & 0 & 1044 & 1083 \\
\hline 2.4 & 9 & 0 & 9 & 0 & 750 & 736 \\
\hline 2.5 & 9 & 0 & 10 & 1 & 881 & 870 \\
\hline
\end{tabular}

Although the results were obtained from a non-dedicated machine, we report the best observed performance from a number of experiments and believe the trends to be valid. Thus with a single thread we see maximum performance from experiment number 1.1 - corresponding to co-location of the thread and memory. Separating the thread and memory by a single R-brick decreases bandwidth (result 1.2) by about $20 \%$. This decreases still further when the thread and memory are separated by two Rbricks (result 1.3). Since the interconnect can support data rates well in excess of the results obtained above, it seems likely that at least part of this bandwidth reduction may be due to contention on the network arising from the other jobs running at the time.

Considering now the results for two threads, if these threads plus their memory are all located on the same C-brick we see only marginal improvement in bandwidth compared to the single thread results, i.e. result 2.1 compared to result 1.1. Moving the threads and memory to different C-bricks (result 2.2) gives a significantly enhanced bandwidth of nearly twice that of a single thread (result 1.1). Interestingly having each thread reference memory on the C-brick of the competing thread (result 2.3) produces nearly the same bandwidth as when each thread is accessing local memory (result 2.2). In experiment 2.4 the threads were both located on the same Cbrick but at the opposite side of the machine to their memory. This produces roughly the same bandwidth as having all threads and memory on the same C-brick (result 2.1). Finally having all threads and memory on different C-bricks is slightly better than having them all on one $\mathrm{C}$-brick, i.e. result 2.5 compared to result 2.1. 
In summary for two threads their appears to be the potential for the available memory bandwidth to vary by a factor of two between the best and worst possible thread and memory placement.

\section{Memory Placement for 2D Heat Diffusion}

The above results clearly show a difference between optimal and non-optimal memory and thread placement. Both cases are, however, extreme examples designed to illustrate these effects. We now consider a slightly more realistic problem - the solution of the Laplace equation on a uniform rectangular lattice with Dirichlet boundary conditions. A typical example of this occurs when calculating the steady state temperature distribution for a metal plate whose edges are held at a fixed temperature. The algorithm proceeds by discretizing the domain of the metal plate and then evaluating a new temperature for each grid point based on an average of the current temperatures of the four surround grid points. Normally the calculation would continue in an iterative fashion until some convergence criteria is reached, however in our benchmark we perform a fixed number of iterations. The algorithm is easily parallelized using a simple domain decomposition in which, for example, on two CPUs all the data points in one half of the domain are assigned to one thread while those in the other half are assigned to the other. Our parallelization was implemented using pthreads.

In a similar vein to the modified STREAM benchmark the threads and the memory corresponding to the grid point they will be updating were selectively placed on different C-bricks. In contrast to the simple STREAM benchmarks, however, updating the data points at the boundary between the two domains requires both threads to retrieve a small amount of data from the adjoining domain. The calculation was run for 25 iterations on a grid size of 1500 by 2048 elements. Results for two threads are given in Table 2. Although the benchmark was again run in a nondedicated environment timing data was collected from a number of runs performed over a number of days. We report the best elapsed time as well as the average elapsed time and standard deviation. The thread and memory placements labeled as "IRIX" were obtained without using any explicit memory or thread placement.

The "Best" results show a difference of about $40 \%$ between the results obtained in experiment 2.2 compared with those from experiment 2.4. It is also not surprising, given the STREAM results, that the fastest performance was obtained when each thread was located on a different C-brick, and the arrays containing their grid points also resided on the same $\mathrm{C}$-brick. It is also interesting that the results obtained without any explicit thread or memory placement are close to the worst. Suggesting that, at very least, a user who blindly uses this system without any thought to thread and data location is likely to achieve far from optimal performance. 
Table 2. Effect of memory and thread placement for parallel 2-D heat diffusion benchmark using 2 threads. Results elapsed times obtained from a non-dedicated 64 SGI O3K with $600 \mathrm{MHz}$ processors

\begin{tabular}{|c|c|c|c|c|c|c|c|}
\hline \multirow{2}{*}{$\begin{array}{l}\text { Exp. } \\
\text { No. }\end{array}$} & \multicolumn{2}{|c|}{ - C-Brick Pair 1 - } & \multicolumn{2}{|c|}{ - C-Brick Pair 2 - } & \multirow{2}{*}{$\begin{array}{l}\text { Best } \\
\text { Sec }\end{array}$} & \multirow{2}{*}{$\begin{array}{c}\text { Average } \\
\text { Sec }\end{array}$} & \multirow{2}{*}{$\begin{array}{l}\text { Std. } \\
\text { Dev. }\end{array}$} \\
\hline & Thread & Memory & Thread & Memory & & & \\
\hline 2.1 & 0 & 0 & 0 & 0 & 3.2 & 3.8 & 0.63 \\
\hline 2.2 & 0 & 0 & 1 & 1 & 3.1 & 3.7 & 0.49 \\
\hline 2.3 & 0 & 1 & 1 & 0 & 4.0 & 4.1 & 0.08 \\
\hline 2.4 & 9 & 0 & 9 & 0 & 4.6 & 5.0 & 0.45 \\
\hline 2.5 & 9 & 0 & 9 & 1 & 4.4 & 5.4 & 0.94 \\
\hline 2.6 & IRIX & IRIX & IRIX & IRIX & 4.3 & 4.7 & 0.23 \\
\hline
\end{tabular}

\section{Conclusions}

We believe that quantitatively studying the effects of NUMA for a range of applications will provide valuable input into the debate on whether and how to extend the OpenMP paradigm to account for NUMA. This work is a first step in that direction. Undertaking NUMA studies requires some means to selectively bind threads or processes to specific hardware resources and to allocate memory at specific physical memory locations. One way to do this on the SGI Origin system has been discussed. Using the SGI we have developed benchmarks that can be used to accurately measure the differential memory latencies and the effects of bandwidth contentions. In both cases we have shown that the difference between good and bad memory/thread placement on the SGI Origin can be quite large (a factor of 2 or more). Moreover, our results have been obtained on a relatively small system with just 64 processors and three NUMA levels, going to a larger machine would be expected to significantly enhance these differences. The importance of these effects for a model application has been discussed. These results also show that simply relying on the $\mathrm{O} / \mathrm{S}$ to perform wise thread and memory placement does not necessarily produce good results. Work is now in progress to extend these investigations to involve larger number of threads, more complicated algorithms and other NUMA architectures.

Acknowledgements. The authors are grateful to Martin Nichols and the SGI staff at the University of Queensland for their assistance in this work. The support of the Australian Partnership in Advanced Computing is gratefully acknowledged. 


\section{References}

1. See http://www.openmp.org

2. A. Basumallik, S-J. Min and R. Eigenmann, Towards OpenMP Execution on Software Distributed Shared Memory Systems, pp 457-468, Int. Workshop on OpenMP: Experiences and Implementations (WOMPEI2002), Lecture Notes in Computer Science \#2327, Springer Verlag, May 2002.

3. Omni OpeMP and SCASH. http://www.pccluster.org

4. D.S. Nikolopoulos, T.S. Papatheodorou, C.D. Polychronopoulos, J. Labarta and E. Ayguadé, Is Data Distribution Necessary in OpenMP, Proc. Supercomputing, Dallas, TX 2000, IEEE Press.

5. J. Bircsak, P. Craig, R. Crowell, Z. Cvetanovic, J. Harris, C.A. Nelson and C.D. Offner, Extending OpenMP for NUMA Machines, Proc. Supercomputing, Dallas TX 2000, IEEE Press.

6. D.S. Nikolopoulos, T.S. Papatheodorou, C.D. Polychronopoulos, J. Labarta and E. Ayguadé, A Case for User-Level Dynamic Page Migration, Proc. of the $14^{\text {th }}$ ACM Int. Conf. on Supercomputing, pp 119-130. Santa Fe, NM, May 2000.

7. See Chapter 8: Tuning for Parallel Processing, Origin 2000 and Onyx 2 Performance Tuning and Optimization Guide (document 0073430 003), available at http://techpubs.sgi.com

8. Tru64 UNIX NUMA Overview, Part Number AA-NUMAG-DE, available at http://www.tru64unix.compaq.com/docs/base_doc/DOCUMENTATION/V51_HTML/NU MA/numa.pdf

9. See http://www.sgi.com/origin/3000/

10. J.R. Mashey, NUMAflex Modular Design Approach - A revolution in Evolution, http://www.cwi.nl/ robertl/mash/numaflex, posted in Aug. 2000 on comp.arc.news

11. See Appendix C, Useful Scripts and Code, Origin 2000 and Onyx2 Performance Tuning and Optimization Guide (document 0073430 003), available at http://techpubs.sgi.com

12. L. McVoy, LMBench - Tools for Performance Analysis, http://www.bitmover.com/lmbench

13. J.D. McCalpin, STREAM: Sustainable Memory Bandwidth in High Performance Computers, http://www.cs.virginia.edu/stream 